\title{
Intrinsic and extrinsic factors relevant to the morphology of the growing cricoid ring after a combined anterior and posterior cricoid split: an experimental study in rabbits
}

\author{
Jim K. Bean*, Henriette L. Verwoerd-Verhoef, \\ Carel D.A. Verwoerd \\ Department of Otorhinolaryngology, Dykzigt Hospital/Erasmus University Rotterdam. Rotterdam. \\ Netherlands
}

(Received 3 August 1993; accepted 12 September 1993)

\begin{abstract}
The effects of a Rethi procedure upon the cricoid is investigated in young rabbits. An anterior and posterior cricoid split carried out upon the larynx of a young rabbit was demonstrated to result in an enlarged cricoid lumen in the adult stage due to an enhancement of both the anterior and posterior transversal diameter of the ring. These changes are ascribed to a release of interlocked stresses in the cricoid cartilage and the action of the cricopharyngeal and cricothyroid muscle.
\end{abstract}

Key words: Cricoid; Rethi procedure; Combined anterior-posterior cricoid split; Cricopharyngeal muscle; Subglottic stenosis

\section{Introduction}

For many decades, surgical trauma inflicted to the soft tissues and the cartilaginous structures of the larynx of a child was supposed to compromise the growth potential. Jackson [9] stated in 1932 that 'the utmost conservatism was fundamental in dealing with the larynx of a child'. It was not until 1956 that Rethi [12] pioneered the concept of a surgical expansion of the stenotic laryngeal lumen by splitting the

* Corresponding author, E.N.T. Department, Dykzigt Hospital, Dr. Molewaterplein 40, 3015 GD Rotterdam, Netherlands. 
cartilaginous skeleton. He proposed dividing the cricoid ring by a median incision anteriorly and posteriorly. Since then, several authors have reported a successful decannulation of young patients with a severe subglottic stenosis treated by a combined anterior and posterior cricoid split [5,7,8,11]. A long-term functional and radiographic evaluation of the expansion of the growing airway after decannulation is not a routine procedure. Therefore, it is not yet known whether such a procedure will interfere in the long run with the growth potentials in the cricoid cartilage. However, Rinne et al. [13] described that 3 out of 10 children, treated with a Rethi operation, developed a gradually progressive subglottic stenosis at puberty without giving details on the cricoid.

Experimental studies concerning the effects of an anterior and posterior cricoid split upon the subglottis have been mainly restricted to adult animals $[6,10,16]$. Only Silver et al. [15] studied the effects of an anterior-posterior cricoid split in young animals during growth. They concluded that this surgical procedure does not result in a diminished growth of the cricoid. However, no data were presented relevant to the (late) effects on the morphological development of the cricoid.

This study aims to investigate the consequences of the combined anterior and posterior cricoid split for the later development of the (divided) cricoid. The larynges of growing rabbits were chosen as an experimental model because various aspects of normal growth and wound healing of the subglottic area were previously described [1-3].

\section{Materials and methods}

Experimental series: 10 young (4-week-old) female New Zealand white rabbits were subjected to a modified Rethi procedure - anterior-posterior cricoid split (APCS) as described later. Their weight at the time of surgery varied from 450 to $600 \mathrm{~g}$. The animals were anaesthetized by intramuscular administration of ketamine hydrochloride $(0.8 \mathrm{ml} / \mathrm{kg}$ body weight) and thiazine hydrochloride $(0.8 \mathrm{ml} / \mathrm{kg}$ body weight). Postoperatively, the animals received a mixture of procain-penicillin and benzathine-penicillin $(0.1 \mathrm{ml} / \mathrm{kg})$ subcutaneously. The rabbits were sacrificed 20 weeks later, in the adult stage. Control series: 20 adult animals sacrificed at the age of 24 weeks.

All larynges were histologically processed; $5 \mu$ thick transverse sections were prepared and stained in a Pas-Alcian blue solution (Fig. 1a). In order to compare the dimensions of the control and operated cricoids, the landmarks B, C, D and E were used (Fig. 1b), according to the morphometrical method described earlier [1]. The morphometrical data - BC, DE, the gap between the anterior stumps and the surface area enclosed by the cricoid ring (cricoid lumen) - were obtained by measuring the histological slides with a Videoplan device.

On both sides the most anterior and most posterior parts of the insertion of the cricopharyngeal muscle (CPM) upon the dorsolateral part of the lamina of the cricoid were chosen to define a line. The angle (CPA) between these two lines was measured in the APCS and control specimens (Fig. 1b).

Modified Rethi procedure (APCS)

Via a midline incision through skin and subcutis and separation of the muscles 

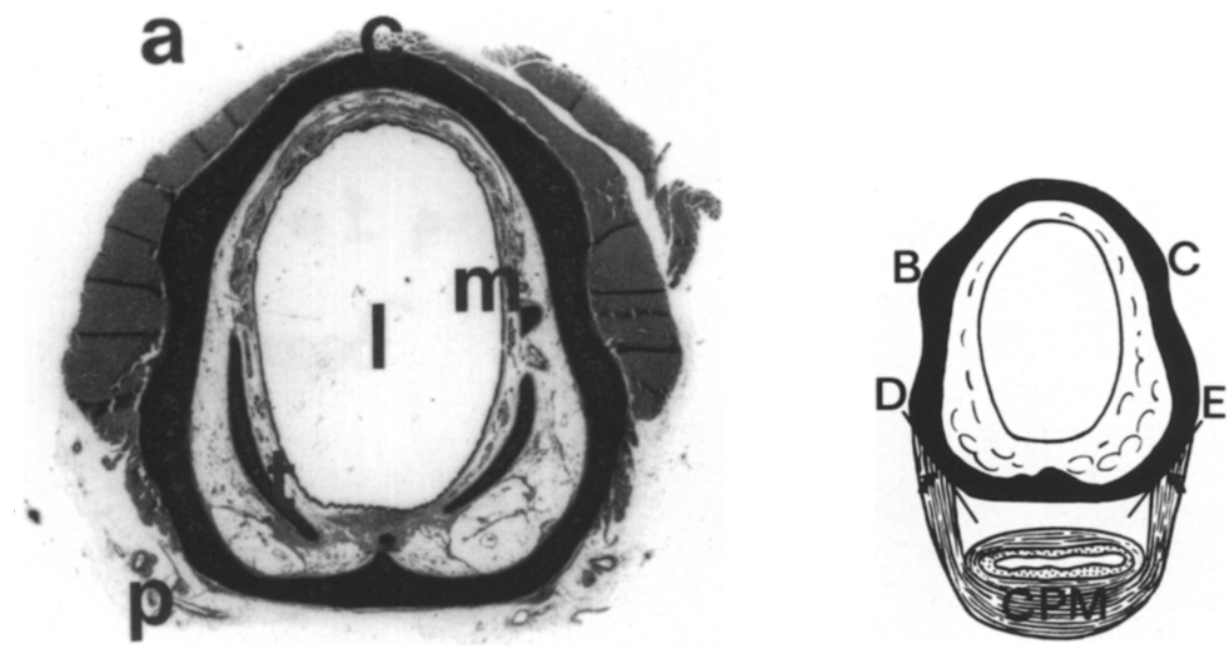

Fig. 1. (a) Transverse histological section of the normal cricoid of an adult rabbit; magn. $\times 10$. c. cricoid cartilage; a, anterior; $p$, posterior; $t$, part of first tracheal ring; $m$, mucosa, with submucosa; $l$, airway lumen. (b) Schematic drawing of (a). B and $C$ are landmarks which indicate the transition between the anterior cricoid arch and the lateral parts of the cricoid ring. D and $E$ indicate the transitional zone between the lateral parts of cricoid ring and lamina of the cricoid. The insertion of the CPM upon the dorsolateral side of the cricoid ring is indicated (arrow). Along these insertion areas two oblique lines which create an angle, are drawn.

overlying the airway, the cricoid is exposed and split in the midline. The split creates an immediate gap as the cut edges recede promptly due to a release of interlocked stresses [18]. The airway lumen is entered via a sagittal incision through the subepithelial layer and epithelium, extending from the first tracheal ring towards the caudal edge of the thyroid cartilage. The lamina of the cricoid is reached through a translaryngeal median incision of the overlying mucosa and submucosal layer on the posterior side. This is followed by a median split of the lamina. Care was taken not to damage the underlying oesophagus. Total longitudinal splitting of the lamina was assured by momentarily pulling the two halves apart. No stents were used. The cervical wound was closed in layers with absorbable sutures.

\section{Results}

\subsection{Morphological observations}

In all specimens of the APCS series, the anterior cricoid gap is widely open (Figs. $2,3)$. The anterior cricoid stumps are rounded and covered with perichondrium. Apposition of new cartilage by this perichondrium is a regular finding (Fig. 4a). There are no signs of necrosis. Collagen fibers can often be observed between both anterior stumps (Fig. 2). Posteriorly, the cut edges of the lamina are approximated and reconnected by collagen fibers or fibrocartilaginous tissue or newly formed hyalin cartilage (Fig. 4b). A slight overlap of the edges was found in 3 specimens (Fig. 3). 


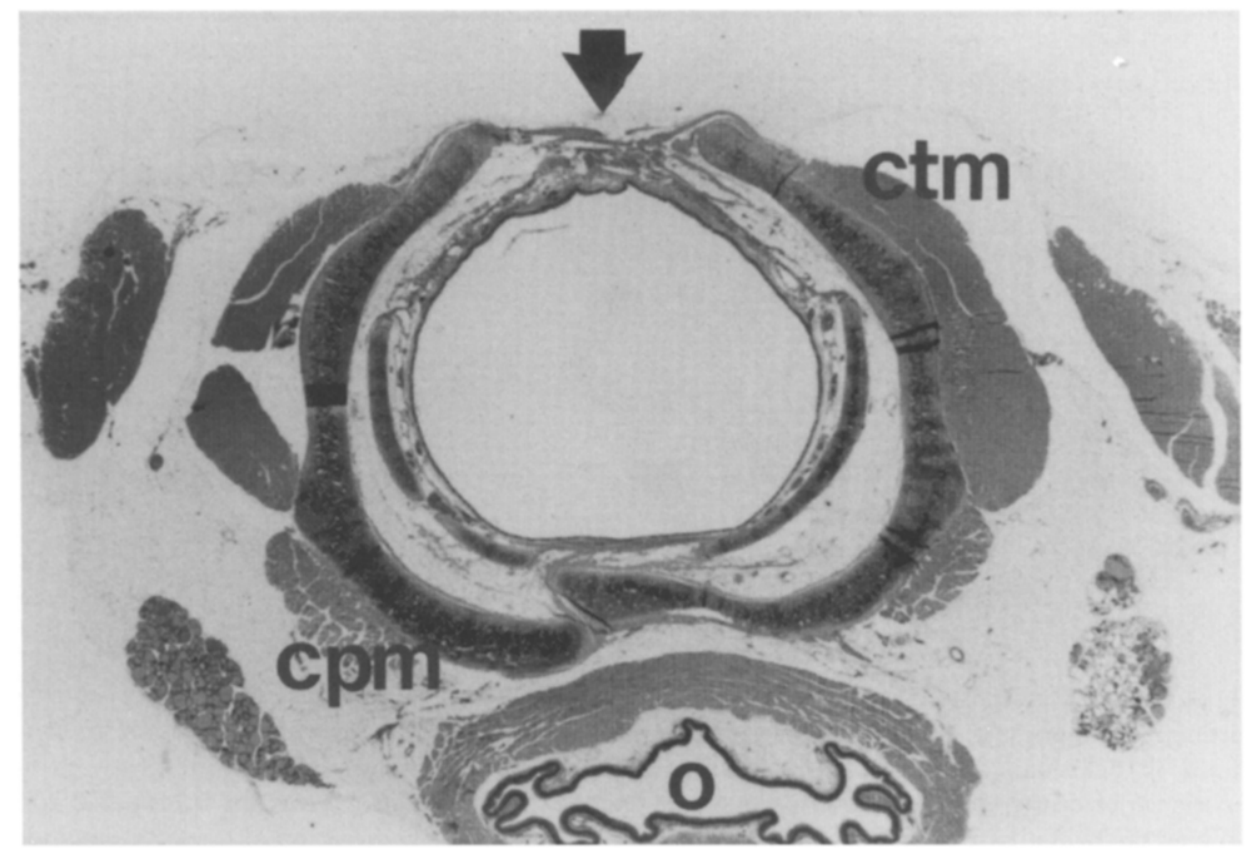

Fig. 2. Histological section of adult cricoid, 20 weeks after an anterior posterior cricoid split. Anterior gap with collagen fihers in between (arrow) and posterior (fibrous) fusion with slight overlap of the posterior stumps. CPM, insertion of cricopharyngeal muscle upon cricoid; CTM, insertion of cricothyroid muscle; $O$, oesophagus with constrictor muscle.

The morphology of the separated halves of the cricoid ring is always abnormal and shows a marked individual variation (Fig. 3). Most of the cricoid segments demonstrate a degree of stretching compared to their normal curvature. The anterior endings are outwardly rotated, contributing to an enlargement of the anterior gap.
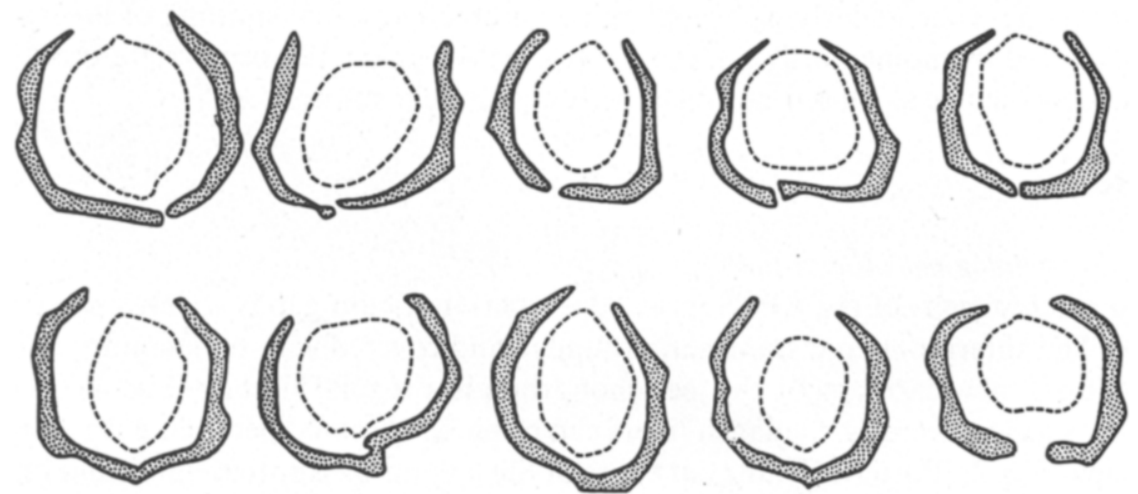

Fig. 3. Schematical drawing of cricoid in APCS series. Anterior and posterior widening of the cricoid ring is observed in all specimens. Lumen of the cricoid ring has changed and has a more circular configuration. 

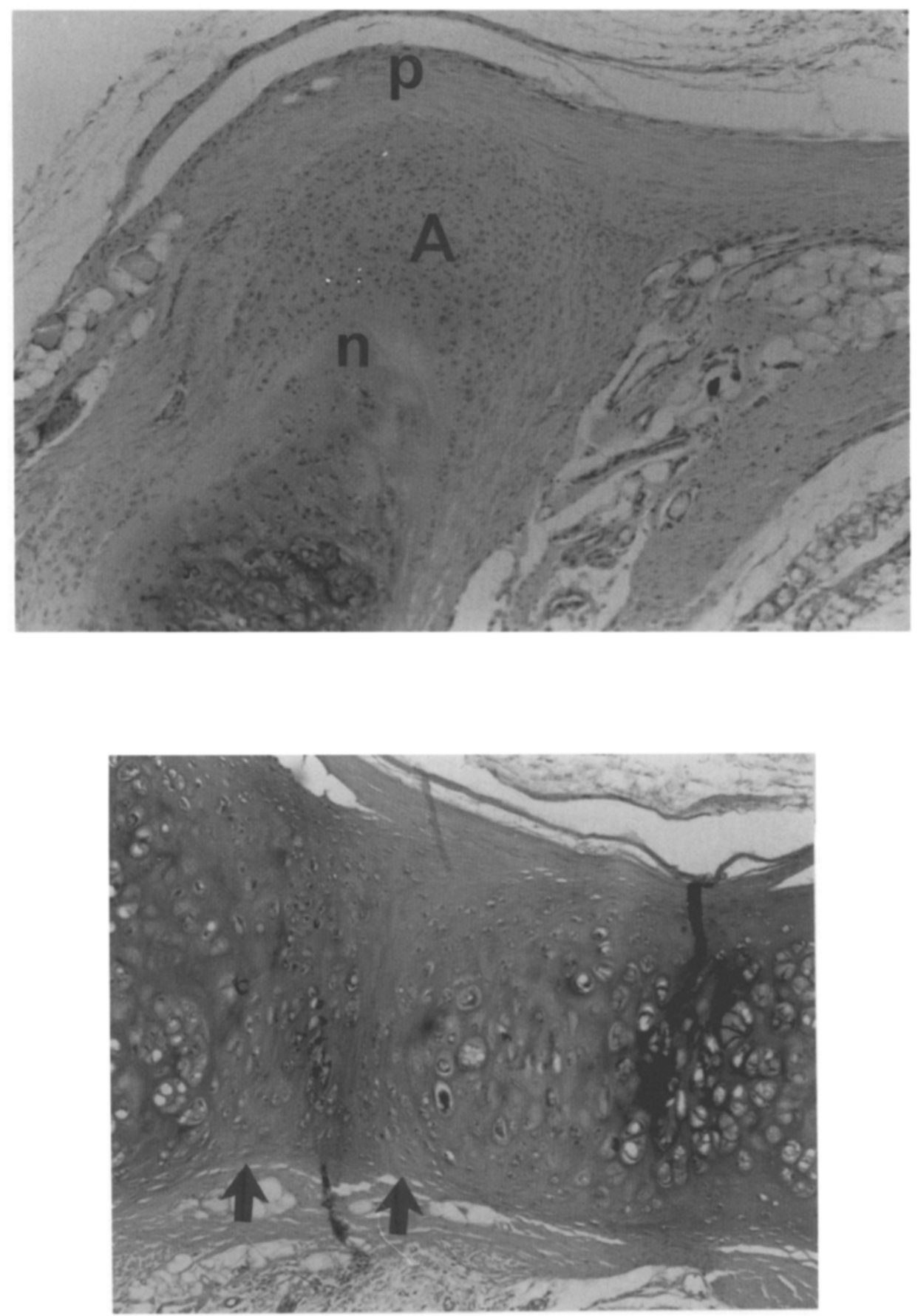

Fig. 4. Detailed view of anterior stump (a) and posterior fusion (b). (a) Thickening of the anterior stump by newly formed cartilage. $p$, perichondrium; a, appositional (newly formed) cartilage; $n$, necrosis. (b) Cartilaginous fusion of the cut edges of the lamina: arrows indicate the transection edges of lamina. 
Finally, the specific pattern of thinner and thicker areas of the cricoid ring, a constant finding in control animals, appeared to be highly irregular or even absent in the APCS series.

At the site of the anterior and posterior incision through the soft tissues lining the airway, the mucosa has completely regenerated and is covered by a normal ciliated cylindrical epithelium. The subepithelial layer might be slightly thickened as a result of the deposition of fibrous tissue and occasionally ectopic cartilage. Inflammatory cells cannot be found at this stage. In 8 out of the 10 specimens the first tracheal ring is almost completely present in sections at the level of the cricoid ring, and in some cases it appeared that this first tracheal ring had been dissected.

\subsection{Morphometrical observations (Table 1)}

For the anterior gap an average of $4.39 \mathrm{~mm}$ was measured (Table 1). Compared to the adult control series, the cricoids in the APCS group show an increased transverse diameter at the levels $\mathrm{B}-\mathrm{C}$ and $\mathrm{D}-\mathrm{E}$. The area enclosed by the interrupted cricoid ring is larger in the APCS group (47.78 $\mathrm{mm}^{2}$, S.D. 7.24) than in the control group (32.68 $\mathrm{mm}^{2}$, S.D. 3.80). The angle (CPA) determined by the lines through the insertion of the CPM on both sides of the cricoid (Fig. 1b) appeared to be significantly larger in the APCS series than in the control specimen.

\section{Discussion}

An anterior and posterior cricoid split (APCS) is demonstrated to induce in young rabbits a specific pattern of abnormal development of the divided cricoid ring, characterized by:

(1) A large persistent gap between the anterior stumps of the cricoid.

(2) Reconnection of the cut edges of the lamina (posterior ends) in most of the specimens.

(3) Stretching of the separated cricoid fragments.

(4) Outward rotation of the cricoid halves (increased CPA, BC and DE).

Table 1

Mean values of the cricoid lumen and the transversal diameter (B-C, D-E), after a combined anterior and posterior cricoid split

\begin{tabular}{lcllll}
\hline & $\begin{array}{l}\text { Cricoid } \\
\text { lumen } \\
\mathrm{mm}^{2}\end{array}$ & $\begin{array}{l}\text { Gap } \\
\mathrm{mm}\end{array}$ & $\begin{array}{l}\text { B-C } \\
\mathrm{mm}\end{array}$ & $\begin{array}{l}\mathrm{D}-\mathrm{E} \\
\mathrm{mm}\end{array}$ & $\begin{array}{l}\text { Angle (CPA) } \\
\text { degrees }\end{array}$ \\
\hline Control & 32.68 & - & 5.19 & 6.07 & 60 \\
S.D. & 3.80 & - & 0.43 & 0.39 & 10 \\
APCS & $47.78^{*}$ & 4.39 & $7.06^{*}$ & $7.43^{*}$ & $93^{*}$ \\
S.D. & 7.24 & 1.61 & 0.87 & 0.73 & 20 \\
\hline
\end{tabular}

*Significantly different with $P<0.05$, Mann-Whitney test. 
These changes result in an increased cricoid lumen cross sectional area. Modifications of the morphological development of the cricoid ring as the result of a single or multiple split have been reported previously [2]. An essential feature of the growing fragments of the cricoid was demonstrated to be a diminished curving of the cartilage. This has been ascribed to the release of interlocked stresses in the cartilage, which are thought to be stronger at the outer than at the inner margin of the cricoid ring [18]. In this respect the longitudinal halves after an APCS react similar to the anterior and posterior segments after a bilateral split [18]. On the other hand, release of interlocked stresses cannot explain the outward rotation of the cricoid halves with a persistence of the anterior gap and reconnection of the posterior stumps. Therefore, the question arises which extrinsic forces could influence the cricoid fragments.

The cricoid is suspended in a three dimensional system of muscles, of which the cricopharyngeal muscle (CPM) and the cricothyroid muscle (CTM) are the most conspicuous. The CPM inserts upon the dorsolateral part of the lamina on both sides and surrounds the pharynx. Contraction of the CPM during swallowing results in a pulling force at the sites of insertion (Fig. 5).

After an APCS this could lead to reapproximation of the posterior cut edges, an outward rotation of the mobile cricoid halves and an enlargement of the anterior gap. As these phenomena are actually observed in the APCS series it may be hypothesized that the action of the CPM contributes to the morphological changes of the longitudinally split cricoid ring.

The insertion of the CTM is found upon the cricoid arch and the anterior edge of the cricoid lamina. Its major action comprises an elevation of the cricoid ring. A relatively small component of this muscle action could be involved in lateral displacement of the separated cricoid halves [14]. Further experimental analysis is needed to elucidate the influence of various laryngeal muscles on cricoid pathology.

It can be concluded, however, that morphological changes of the cricoid after an APCS are the result of an intrinsic factor (release of interlocked stresses in the cartilage) and an extrinsic factor (muscle action). Splitting of the cricoid means interrup-
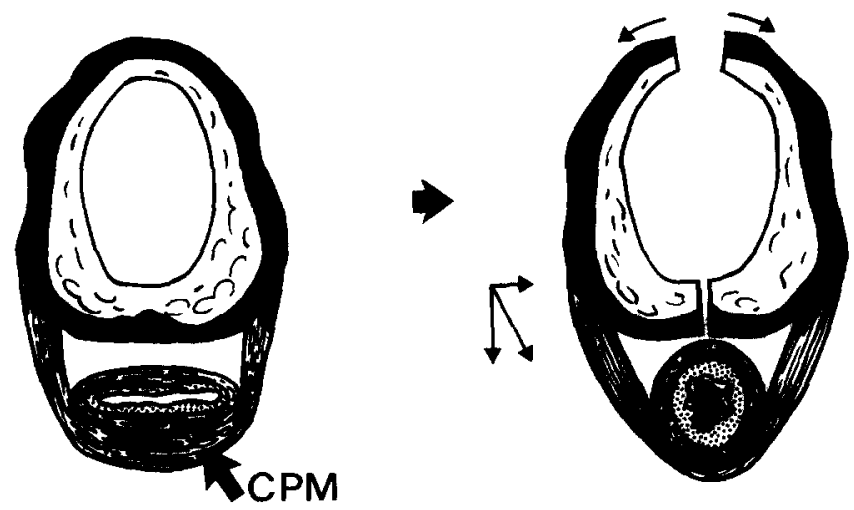

Fig. 5. Schematic drawing of the CPM and the insertion upon the cricoid ring as seen in the transverse plane. The contracting CPM exerts a tilting force upon the lateral halves of the cricoid. divided by an APCS. 
tion of the ring and interference with the balanced forces of the muscular system in which the cricoid is suspended, both with consequences for the future morphological development.

Previous authors $[10,15]$, studying various splits in animal experiments, did not comment on the morphological changes of the cricoid, as reported here. They focused on the dimensions of the subglottic airway [10] and the cross-sectional area of the cartilage of the cricoid ring. Strome et al. [16] and Zalzal et al. [19] did report an unstable apposition of the posterior stumps in adult dogs contrary to the fibrous and cartilaginous connections in our experiments. This difference could be related to a difference in species (dog-rabbit) or age (young-adult) at the time of surgery. Recently, a decrease of wound healing capacities of the cricoid cartilage was demonstrated with increase in age [4]. This could explain the lesser stability of the posterior edges in adult experimental animals compared to young animals. The longitudinal incisions in the soft tissue lining both posteriorly and anteriorly showed in our series an excellent wound healing without the formation of a stenosis. Prolonged inflammatory processes seem the most probable reason for scar formation and a soft tissue stenosis [3]. To what extent the increase of the cricoid 'lumen' results in an increase of the subglottic airway cannot be concluded from these data at hand as the cross-sectional airway lumen at the level of the cricoid appeared to be defined in most specimens by the first tracheal ring, trapped within the cricoid. It is possible that widening of the cricoid lumen promotes this upward displacement (trapping) of the first tracheal ring.

\section{Conclusions}

(1) A combined anterior-posterior cricoid split in young rabbits results in a specific pattern of maldevelopment of the cricoid ring.

(2) The anterior gap after a combined anterior-posterior cricoid split is larger compared to the gap after a solitary anterior cricoid split because of the tilting action of the CPM upon the posterior part of the cricoid, and the release of interlocked stresses in the cricoid segments.

\section{References}

1 Adriaansen, F.C.P.M., Verwoerd-Verhoef, H.L., van der Heul, R.O. and Verwoerd, C.D.A. (1986) A morphometric study of the growth of the subglottis after endolaryngeal trauma. Int. J. Pediatr. Otorhinolaryngol. 12, 217-226.

2 Adriaansen, F.C.P.M., Verwoerd-Verhoef, H.L., van der Heul, R.O. and Verwoerd, C.D.A. (1988) Histologic study of the growth of the subglottis after interruption of the circular structure of the cricoid. Otorhinolaryngology 50, 94-102.

3 Adriaansen, F.C.P.M., Verwoerd-Verhoef, H.L., van der Heul, R.O. and Verwoerd, C.D.A. (1988) Differential effects of endolaryngeal trauma upon the growth of the subglottis. Int. J. Pediatr. Otorhinolaryngol. 15, 163-171.

4 Bean, J.K., Verwoerd-Verhoef, H.L., Adriaansen, F.C.P.M. and Verwoerd, C.D.A. (1992) The influence of ageing on wound healing of the cricoid. Acta Otolaryngol. (Stockh.) 112, 362-365.

5 Cotton, R.T. (1987) The management and prevention of subglottic stenosis in infants and children. Adv. Otolaryngol. Head Neck Surg. 1, 24l-260.

6 Cotton, R.T. (1991) The problem of pediatric laryngotracheal stenosis: a clinical and experimental 
study on the efficacy of autogenous cartilaginous grafts placed between the vertically divided halves of the posterior lamina of the cricoid cartilage. Laryngoscope 101, 1-34.

7 Crysdale, W.S. (1982) Surgical correction of subglottic stenosis in children. J, Otolaryngol. 11. 209-213.

8 Grahne, B. (1971) Operative treatment of severe chronic traumatic laryngeal stenosis in infants up to three years old. Acta Otol. 72, 134-137.

9 Jackson, C. (1932) Laryngeal stenosis: growth of the larynx as a factor in treatment. Laryngoscope 2. $887-889$.

10 MacArthur, C.J. and Senders, G.W. (1992) Variations of the anterior cricoid split: the effect on airway cross-sectional area in the rabbit. Laryngoscope 102, 250-255.

11 Narcy, P., Contencin, P., Fligny, I. and Francoise, M. (1990) Surgical treatment for laryngotracheal stenosis in pediatric patients. Arch. Otolaryngol. Head Neck Surg. 116, 1047-1050.

12 Rethi, A. (1956) An operation for cicatricial stenosis of the larynx. J. Laryngol. 70, 283-293.

13 Rinne, J., Grahne, B. and Sovijarvi, A.R. (1985) Long term results after surgical treatment of laryngeal stenosis in small children. Int. J. Pediatr. Otorhinolaryngol. 10, 213-220.

14 Senders, G.W. and Eisele, P. (1989) Independent effects of denervating the cricothyroid muscle and stenting on the anterior cricoid split:canine model. Int. J. Pediatr. Otorhinolaryngol. 17, 213-224.

15 Silver, F.M., Meyer, C.M., III and Cotton, R.T. (1993) Lateral division of the rabbit cricoid cartilage: its effect on cartilage growth. Otolaryngol. Head Neck Surg. 103, 63-69.

16 Strome, M., Norris. C.M., Jr and Joseph. M.P. (1982) An assessment of grafts in the posterior cricoid lamina. Laryngoscope 92, 1120-1125.

17 Verwoerd, C.D.A., Verwoerd-Verhoef, H.L. and Meeuwis, C.A. (1989) Stress and wound healing of the cartilaginous nasal septum. Acta Otolaryngol. (Stockh.) 107, 441-445.

18 Verwoerd, C.D.A., Bean, J.K., Adriaansen, F.C.P.M. and Verwoerd-Verhoef, H.I. (1991) Trauma of the cricoid cartilage and interlocked stress. Acta Otolaryngol. (Stockh.) 111, 403-409.

19 Zalzal, G.H. and Deutsch, E. (1991) External fixation using microplates after laryngeal expansion surgery; an animal study. Arch. Otolaryngol. Head Neck Surg. 117. 155-159. 